(C) 2008 IEEE. Personal use of this material is permitted. Permission from IEEE must be obtained for all other uses, in any current or future media, including reprinting/republishing this material for advertising or promotional purposes, creating new collective works, for resale or redistribution to servers or lists, or reuse of any copyrighted component of this work in other works.

https://dx.doi.org/10.1109/UKSIM.2008.43 


\title{
Stochastic Analysis of the Packet-Pair Bandwidth Probing Event under Heterogeneous Cross-Traffic
}

\author{
M. J. Tunnicliffe \\ Faculty of Computing, Information Systems and Mathematics, Kingston University, \\ Kingston-on-Thames, Surrey, KT1 2EE. +20-85472000+62674 M.J.Tunnicliffe@king.ac.uk
}

\begin{abstract}
An analytical model of packet-pair bandwidthprobing under heterogeneous traffic is compared with a discrete-event simulation. The arrival of each packet-type is governed by an independent Poisson process, such that the aggregate distribution is approximately Gaussian. The waiting-time can be resolved into two components: A transient component representing the emptying process, and an equilibrium component representing the return to a steady-state distribution. The simulated waiting-time and dispersion characteristics agree closely the model's predictions.
\end{abstract}

\section{Introduction}

If a network path has a capacity $l$ bits/s, and carries cross-traffic $c$ bits/s, then the utilization $\rho=c / l$ and the bandwidth available for new users $A B=l-c$. Reliable $A B$ estimates are useful to network clients who require a minimum bandwidth for real-time applications, and system administrators for achieving optimal performance [1].

The packet-pair technique of Melander et al. [2] gauges available bandwidth in terms the dispersion between probe-packets transmitted in closely-spaced pairs. In the original model, cross-traffic was assumed to behave as a continuous "fluid", such that the individual packet service time was zero. If the first packet in a pair contains $S_{p}$ bits, it blocks the channel for $S_{p} / l$ seconds, creating a backlog of $c \cdot S_{p} / l$ bits. If the second packet arrives before this backlog clears, it is delayed by the residual waiting time and the time separation between the packet deliveries increases. By plotting the dispersion ratio (i.e. the ratio of output to input packet separation) against the probing rate, $l, c$ and thus the available bandwidth can (in principle) be determined.
An earlier paper [5] presented a model of the packet-pair probing event, which agreed closely with the results of a discrete-event simulation for both single and multi-hop network paths. However, this model assumed that the network cross-traffic was composed of identical uniform-sized packets. The current paper extends the model to cover more realistic traffic, composed of different sized packets.

\section{The Packet-Pair Probing Event}

\subsection{The Fluid-Traffic Model}

This analysis concentrates on the "tight link" of a network path, i.e. the link with the smallest $A B$ which dictates the overall path capacity. The derivation below differs from the original [2] in that individual packet arrivals are considered in the time domain. If the first packet in a pair (\#1) arrives at the instant $t=0$, the buffer suddenly acquires $S_{p}$ bits, which complete service at time $t=S_{p} / l$. Meanwhile cross-traffic arrives at $c$ bits/s, so the subsequent waiting-time ${ }^{1}$ profile is $w(t)=\max \left(S_{p} / l-(1-c / l) t, 0\right)$. If Packet \#2 arrives at time $t=\Delta_{i n}$, then it leaves the server when $t=w\left(\Delta_{i n}\right)+S_{p} / l$. It can be seen from Figure 1 that $S_{p} / l+\Delta_{\text {out }}=\Delta_{\text {in }}+w\left(\Delta_{\text {in }}\right)+S_{p} / l$, which can be re-arranged to obtain the dispersion ratio:

$$
D=\frac{\Delta_{\text {out }}}{\Delta_{\text {in }}}=1+\frac{w\left(\Delta_{\text {in }}\right)}{\Delta_{\text {in }}} .
$$

since $w\left(\Delta_{i n}\right)=\max \left(S_{p} / l-(1-c / l) \Delta_{i n}, 0\right)$, it is easy to show that:

1 "Virtual Waiting Time" (VWT) $w(t)$ is the time packet arriving at time $t$ takes to reach the server. Here it was gauged by measuring the sojourn times of "virtual" (zero size) packets. 


$$
D=\max \left(\frac{r}{l}+\frac{c}{l}, 1\right)
$$

where $r$ is the "probing rate", given by $S_{p} / \Delta_{\text {in }}$ bits/s. In principle, the graph of $D$ against $r$ (Figure 2) remains flat at $D=1$ for all $r \leq l-c$ (the effective bandwidth), and increases linearly with a slope $1 / l$ for all $r>l-c$. The values of $l$ and $c$ can therefore be determined by linear regression.

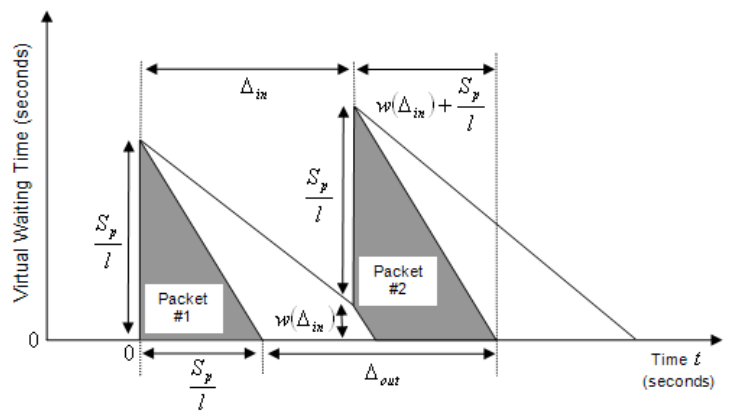

Figure 1. Virtual waiting-time profile during the packet-pair arrival under fluidic cross-traffic.

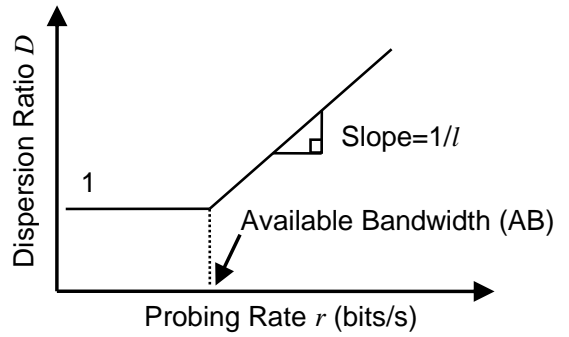

Figure 2. Ideal graph of dispersion ratio vs. probing rate for one congestible link.

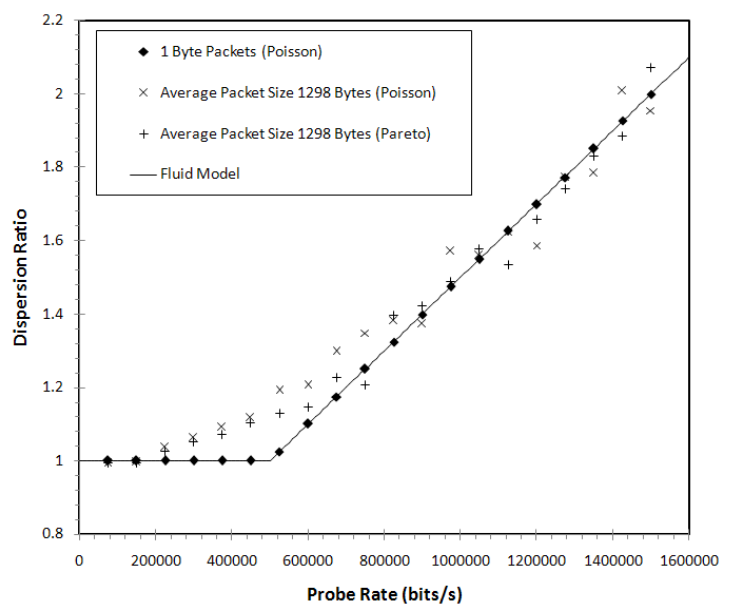

Figure 3. Fluid model and simulation data obtained using various traffic processes. (Server rate $1 \mathrm{Mbit} / \mathrm{s}$, cross traffic $500 \mathrm{kbit} / \mathrm{s}$, available bandwidth $500 \mathrm{kbit} / \mathrm{s}$, probe packets 1000 bytes.)

\subsection{Limitations of the Fluid Model}

Figure 3 compares the model with simulation data obtained using three cross-traffic scenarios. While the 1-byte packets ${ }^{2}$ (which are of negligible size compared to the 1000-byte probe packets) conform to the fluid model, the dispersions obtained using larger variable-size packets tend to be greater than the model's predictions, especially when the probe-rate is close to the available bandwidth (500kbit/s). This is a well-documented effect known as "probing bias" [3], which causes an underprediction of the available bandwidth and an overprediction of the link capacity.

This problem has been studied at a deterministic "sample-path" level [3] and using probabilistic packet-arrival models [4,5]. Park et al. [4] used an exact model of $\mathrm{M} / \mathrm{D} / 1$ queuing dynamics, while Tunnicliffe et al. [5] developed simpler (though nonetheless accurate) approximation.

However, both these models assume that the cross-traffic is Poisson and composed of uniformsize packets. Here we develop an extended version for heterogeneous traffic composed of different sized packets, and compare the predictions with simulation data.

\section{Approximate Stochastic Model}

\subsection{Modeling Heterogeneous Traffic}

For the purposes of this paper, cross-traffic will be assumed to be highly "modal", i.e. composed of a finite number $P$ of independent arrival processes with their own characteristic packet-sizes $S_{1}, S_{2} \ldots . S_{P}$ bits. Let $\beta_{i}$ be the proportion of packets which are of size $S_{i}$, and $\alpha_{i}$ be the proportion of the total traffic (in bits/s) which consists of packets of size $S_{i}$. The quantities $\alpha_{i}$ and $\beta_{i}$ are related by the formulae:

$$
\alpha_{i}=\beta_{i} S_{i} / \sum_{j=1}^{P} \beta_{j} S_{j}, \beta_{i}=\frac{\alpha_{i}}{S_{i}} / \sum_{j=1}^{P} \frac{\alpha_{j}}{S_{j}} .
$$

Table I shows the three packet-size distributions used in this work. Profile 1 was borrowed from [6], while Profiles 2 and 3 were arbitrarily chosen.

2 The simulation software (written in $\mathrm{C}++$ ) is not constrained to the limitations of IP, whose packets are typically $46-1500$ bytes for the Ethernet protocol. 
Table I: The three packet-size distributions

\begin{tabular}{|c|c|c|}
\hline \multicolumn{3}{|c|}{ Profile 1 ( $S_{\text {eff }}=1298$ bytes) } \\
\hline $\begin{array}{c}\text { Packet Size } \\
\text { (bytes) }\end{array}$ & $\begin{array}{c}\text { \% of Total } \\
\text { Traffic }\end{array}$ & $\begin{array}{c}\text { \% of Total } \\
\text { Packets }\end{array}$ \\
\hline 60 & 4.77 & 46 \\
\hline 148 & 2.81 & 11 \\
\hline 500 & 9.50 & 11 \\
\hline 1500 & 82.92 & 32 \\
\hline \multicolumn{3}{|c|}{ Profile 2 ( $S_{\text {eff }}=639$ bytes) } \\
\hline $\begin{array}{c}\text { Packet Size } \\
\text { (bytes) }\end{array}$ & $\begin{array}{c}\text { \% of Total } \\
\text { Traffic }\end{array}$ & $\begin{array}{c}\text { \% of Total } \\
\text { Packets }\end{array}$ \\
\hline 50 & 14.88 & 60 \\
\hline 108 & 10.71 & 20 \\
\hline 500 & 24.80 & 10 \\
\hline 1000 & 49.60 & 10 \\
\hline \multicolumn{3}{|c|}{ Profile 3 ( $S_{\text {eff }}=2218$ bytes) } \\
\hline $\begin{array}{c}\text { Packet Size } \\
\text { (bytes) }\end{array}$ & $\begin{array}{c}\text { \% of Total } \\
\text { Traffic }\end{array}$ & \% of Total \\
\hline 500 & 2.56 & 10 \\
\hline 1000 & 5.13 & 10 \\
\hline 1500 & 15.38 & 20 \\
\hline 2500 & 76.92 & 60 \\
\hline \multicolumn{2}{|c|}{}
\end{tabular}

\subsection{Equilibrium Queue Model}

Now if all the arrival processes are assumed to be Poisson, the bottleneck node may be considered an M/G/1 queue, whose average equilibrium behavior can be modeled using the PollaczekKhintchine (P-K) equation [7]. According this formula, the mean equilibrium waiting time is:

$$
\bar{w}=E(w)=\frac{\lambda E\left(t_{s}^{2}\right)}{2(1-\rho)}
$$

where the packet service time $t_{s}=S / l$ and $\lambda$ is the aggregate packet arrival rate $c \sum_{j=1 . . P} \alpha_{j} / S_{j}$. Thus

$$
\bar{w}=\frac{\lambda E\left(S^{2}\right)}{2 l(l-c)}=\left(\sum_{j=1}^{P} \frac{\alpha_{j}}{S_{j}}\right) \frac{c}{2 l(l-c)} \cdot \sum_{i=1}^{P} \beta_{i} S_{i}^{2}
$$

and using Eqn.(3) to eliminate $\beta_{i}$ yields

$$
\bar{w}=\frac{c}{2 l(l-c)} \cdot \sum_{i=1}^{P} \alpha_{i} S_{i}
$$

The quantity $\Sigma_{i=1 . . P} \alpha_{i} S_{i}$ can be regarded as the "effective packet size" $S_{\text {eff }}$, and is computed for all three packet-size distributions in Table I.
The variance of the waiting time is somewhat more problematic: Exact models exist for the M/D/1 queuing distribution (which could be applied to the M/G/1 system by analogy) but these require some detailed numerical computation.

According to the Heavy Traffic Approximation (HTA), the cumulative queue-size distribution is approximately exponential [8], suggesting that the mean and standard deviation should be approximately equal. This observation was utilized in an earlier paper [5], where (combined with the other approximations in the model) it yielded fairly reasonable results. However, Figure 4(a) shows that the values only truly converge under very high utilization $(\rho \geq 0.8)$, and elsewhere the mean provides a significant under-prediction of the standard deviation.

(a)

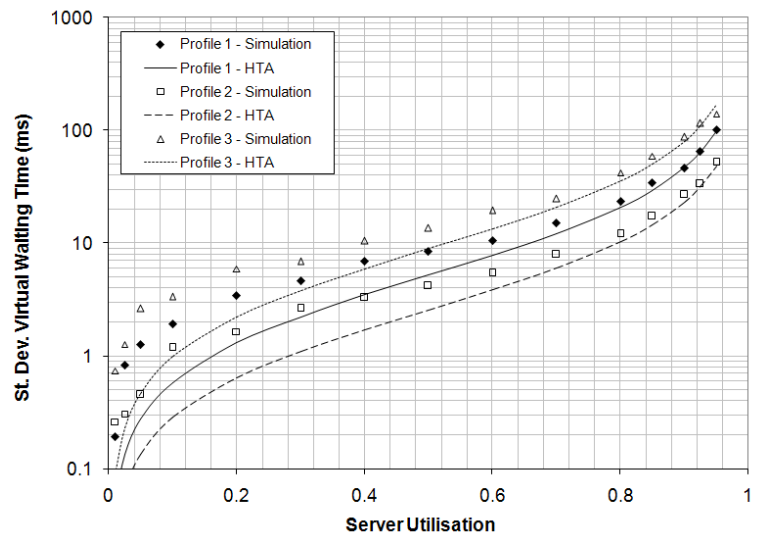

(b)

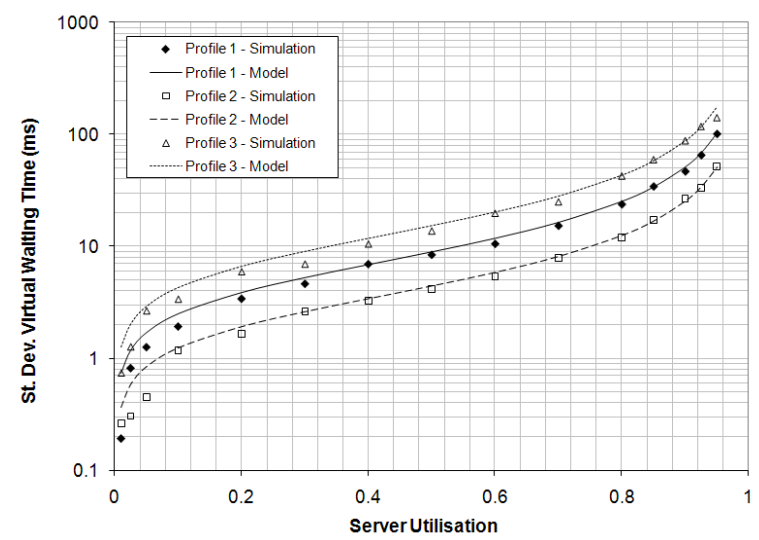

Figure 4. Standard deviation VWT under equilibrium conditions. (a) Simulated standard deviation compared with the Heavy Traffic Approximation (HTA). (b) Simulated standard deviation compared with hybrid deltafunction/HTA model. (Server speed was 1Mbit/s in all cases.) 
In order to achieve a better approximation, we observe that for lower utilizations an arriving packet has a chance $1-\rho$ of finding the buffer empty, and thus experiencing no waiting-time. This component of the waiting-time PDF can be represented by a delta function at $t=0$, with a magnitude $1-\rho$. If an exponential function is assumed for all finite queuesizes, the waiting-time PDF becomes:

$$
f(w)=(1-\rho) \cdot \delta(w)+\frac{\rho^{2}}{\bar{w}} \cdot \exp \left(-\frac{\rho w}{\bar{w}}\right)
$$

and the standard deviation can thus be computed:

$$
\sigma_{w}=\sqrt{E\left(w^{2}\right)-\bar{w}^{2}}=\bar{w} \sqrt{\frac{2}{\rho}-1}
$$

Figure 4(b) compares this with the simulated results for all three packet-size distributions. There is now a slight tendency to over-predict $\sigma_{w}$, but this is only significant under very low utilizations, where the equilibrium waiting-time could for most practical purposes be ignored.

\subsection{Transient Queue Behavior}

At the instant the first probe-packet arrives $(t=0)$, the buffer contains $S_{p}+\bar{w} l$ bits with a standard deviation $\sigma_{w} l$ bits. For $t \geq 0$, two competing processes occur: the backlog is cleared by the server and additional cross-traffic arrives. Using the arrival model developed earlier, the number of packets of size $S_{i}$ to arrive by time $t$ must have a mean $\alpha_{i} c t / S_{i}$ and variance $\alpha_{i} c t / S_{i}$. (A Poisson distribution's variance is equal to its mean.) Thus the mean total arrived bits is $\Sigma_{i=1 . . P} \alpha_{i} c t=c t$ variance $\Sigma_{i=1 . . P} \alpha_{i} c S_{i} t=c S_{e f f} t$ and (before there is any significant chance of the queue emptying completely) the mean number of stored bits is $S_{p}+\bar{w} l-(l-c) t$ with variance $c S_{\text {eff }} t+\sigma_{w}{ }^{2} l^{2}$. If the distribution is approximated by a Gaussian, the waiting-time PDF is given by:

$$
\begin{aligned}
f_{t}(w)=\frac{l}{\sqrt{2 \pi\left(c S_{\text {eff }}+\sigma_{w}{ }^{2} l^{2}\right)}} \\
\cdot \exp \left[-\frac{\left(w-S_{p}-\bar{w} l+(l-c) t\right)^{2}}{2\left(c S_{\text {eff }} t+\sigma_{w}{ }^{2} l^{2}\right)}\right]
\end{aligned}
$$

However, as this applies only for $w \geq 0$ (the queue cannot empty below zero), the resulting contribution to the mean waiting-time must be

$$
\begin{gathered}
\bar{w}_{\text {trans }}(t)=\int_{0}^{\infty} w f_{t}(w) d w \\
=\frac{1}{l} \sqrt{\frac{c S_{\text {eff }} t+\sigma_{w}{ }^{2} l^{2}}{2 \pi}} \exp \left[-\frac{\left(S_{p}+\bar{w} l-(l-c) t\right)^{2}}{2\left(c S_{\text {eff }} t+\sigma_{w}{ }^{2} l^{2}\right)}\right] \\
+\frac{S_{p}+\bar{w} l-(l-c) t}{2 l}\left[1+e r f\left(\frac{S_{p}+\bar{w} l-(l-c) t}{\sqrt{2\left(c S_{\text {eff }} t+\sigma_{w}{ }^{2} l^{2}\right)}}\right)\right]
\end{gathered}
$$

The $n<0$ portion of the Gaussian distribution (Eqn.9) represents the set of possibilities in which the queue has already completely emptied and is recovering its equilibrium state (see Figure 5). Although equilibrium is in reality achieved gradually, for the purposes of the model we will assume that it occurs abruptly $t_{e q}$ seconds (the "effective equilibrium time") after $w$ reaches zero. Thus the waiting-time contribution from equilibrium recovery for $t>t_{e q}$ is given by:

$$
\begin{aligned}
& \bar{w}_{e q}(t)=\bar{w} \int_{-\infty}^{0} f_{t-t_{e q}}(w) d w \\
& =\frac{\bar{w}}{2}\left[1-e r f\left(\frac{S_{p}+\bar{w} l-(l-c)\left(t-t_{e q}\right)}{\sqrt{2\left(c S_{e f f}\left(t-t_{e q}\right)+\sigma_{w}{ }^{2} l^{2}\right)}}\right)\right]
\end{aligned}
$$

(for all $t>t_{e q}$ ) and the overall mean virtual waiting time becomes:

$$
\bar{w}(t)=\left\{\begin{array}{cl}
\bar{w}_{\text {trans }}(t) ; & t \leq t_{e q} \\
\bar{w}_{\text {trans }}(t)+\bar{w}_{e q}(t) ; & t>t_{e q}
\end{array} .\right.
$$

It is convenient to define $t_{e q}$ as the time taken for the mean waiting time of an initially empty queue to reach half its equilibrium value. For the $\mathrm{M} / \mathrm{D} / 1$ system, this was empirically found to follow the equation

$$
t_{e q}=S_{c} /\left(k_{1} l-k_{2} c\right)
$$

where $k_{1}=3.22 \mathrm{~s}^{-1}, k_{2}=3.89 \mathrm{~s}^{-1}$ and $S_{c}$ is the constant packet-size [5]. Figure 6 shows that the equation approximately holds for the three traffic 
profiles in Table I (setting $S_{c}=S_{\text {eff }}$ ) for utilizations up to about 0.75 . (For utilizations above 0.75 , the residual bandwidth is likely to be of poor quality anyway, and of limited use to real-time applications.) Figure 7 shows some simulated mean virtual waiting time profiles (averaged over 1000 simulations), compared with the model predictions.

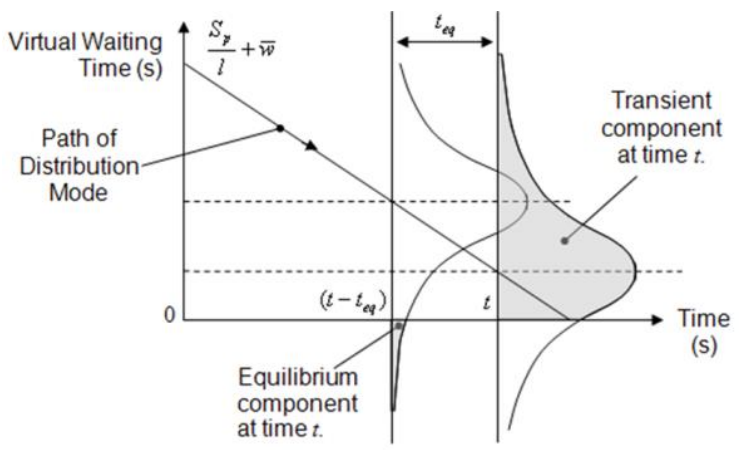

Figure 5. Representation of the Gaussian waiting-time PDF's during buffer emptying.

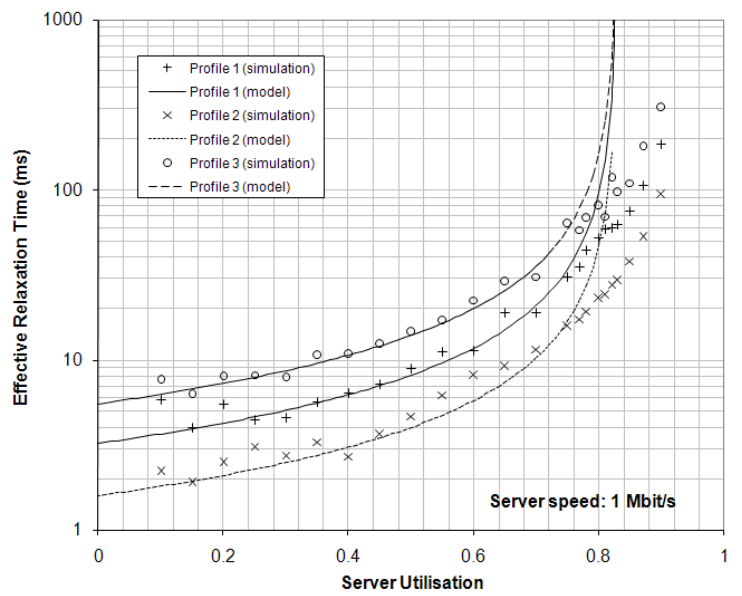

Figure 6. Effective relaxation time as a function of server utilization.

\subsection{Packet Pair Dispersion}

It is simple to map Eqn.12 to the mean interpacket dispersion ratio $D$ : It can be seen from Figure 8 that $\bar{w}+S_{p} / l+\Delta_{\text {out }}=\Delta_{\text {in }}+\bar{w}\left(\Delta_{\text {in }}\right)+S_{p} / l$, so

$$
D=\frac{\Delta_{\text {out }}}{\Delta_{\text {in }}}=1+\frac{\bar{w}\left(\Delta_{\text {in }}\right)-\bar{w}}{\Delta_{\text {in }}}
$$

where $\Delta_{i n}=S_{p} / r$. Figure 9 compares the mean $D$ vs. $r$ characteristics obtained using this model with the results of simulation, showing a close agreement between the two. (a) Traffic Profile 1

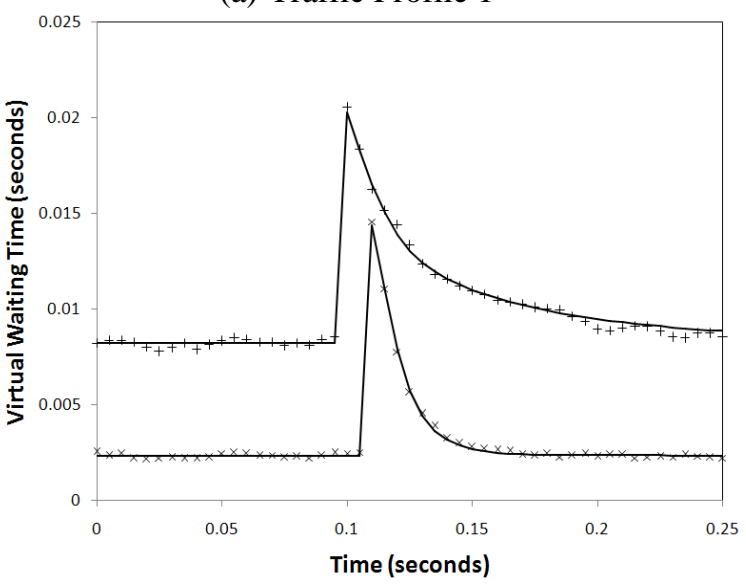

(b) Traffic Profile 2

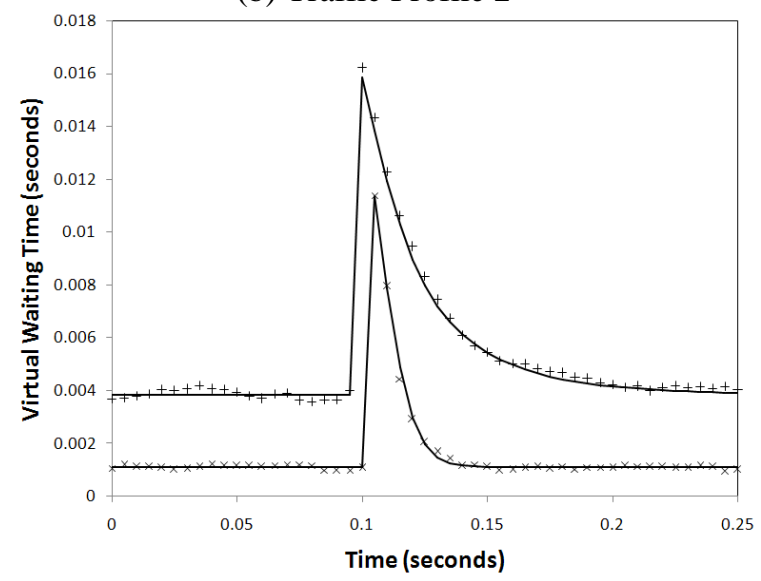

(c) Traffic Profile 3

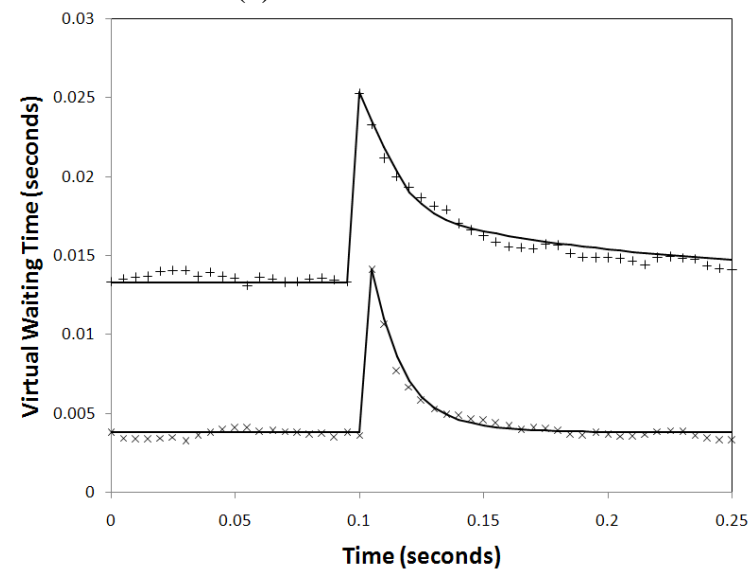

Figure 7. Mean VWT profiles obtained using the three traffic profiles of Table I during the passage of a 1500-byte probe packet. The $x$ 's represent $\rho=0.3$ and the +'s 0.6. Solid lines indicate the predictions of Eqn.12. (Server speed was $1 \mathrm{Mbit} / \mathrm{s}$.) 


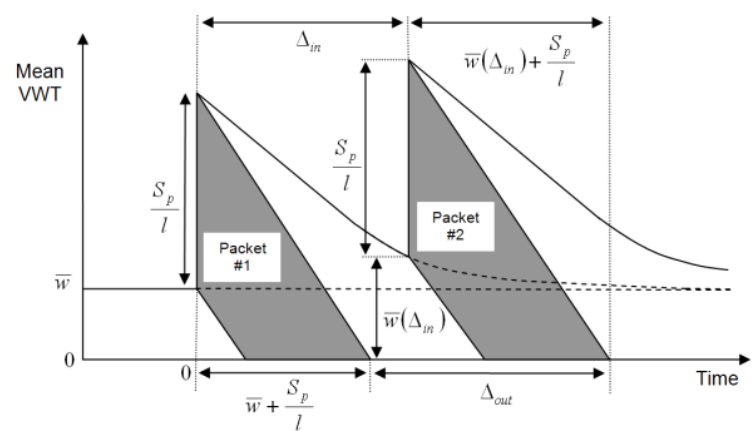

Figure 8. Packet-pair under discrete traffic.

(a) Traffic Profile 1

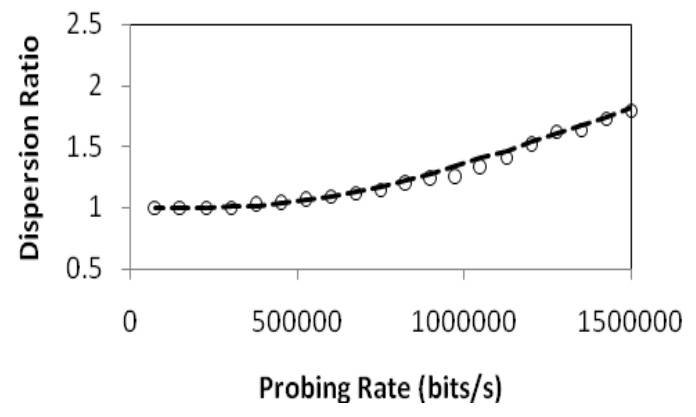

(b) Traffic Profile 2

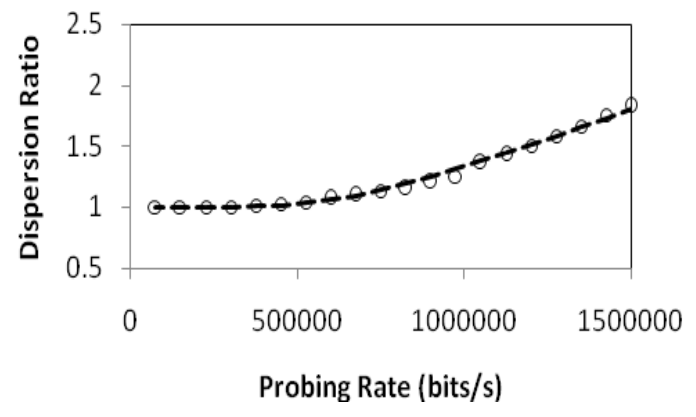

(c) Traffic Profile 3

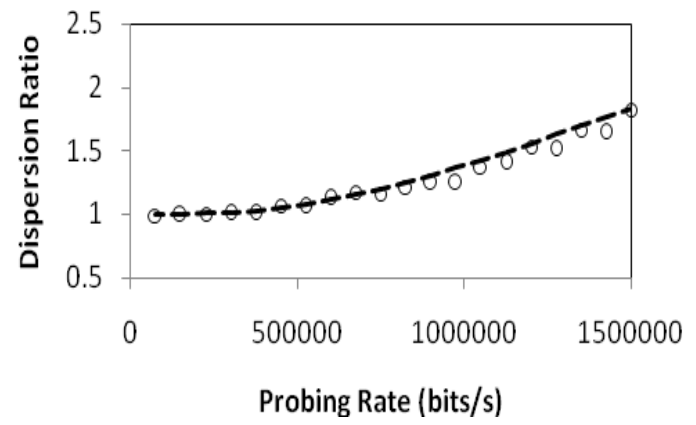

Figure 9. Simulated dispersion profiles (discrete points) compared with analytical predictions (broken lines). The server rate was $1 \mathrm{Mbit} / \mathrm{s}$ and the available bandwidth $700 \mathrm{kbit} / \mathrm{s}$.

\section{Conclusions}

The model previously developed for the M/D/1 system [5] has been extended to cover a more generalized traffic model, and the predictions agree closely with the results of discrete-event simulation. However, the arrival process is still assumed to be Poisson, while real-world traffic is often better represented by long-range-dependent Pareto activity [9]. Part of the model relies on an empirical relationship which breaks down under very high utilization: The model ceases to apply rigidly when $\rho>0.75$. However, connections under such conditions would be likely to experience high latency, jitter, and even data loss, making them unsuitable for most real-time applications.

Future work will ultimately focus on the reverseuse of this model, i.e. determining the bottleneck capacity and cross-traffic from experimental data, and thus monitoring the effective bandwidth.

\section{References}

[1] Crovella,M, Krishnamurthy,B, "Internet Measurement: Infrastructure, Traffic and Applications", Wiley, 2006, p.127-36.

[2] Melander,B Björkman,M, Gunningberg,P, “A New End-to-End Probing and Analysis Method for Traffic Estimating Bandwidth Bottlenecks", Proc. IEEE Globecom'00, San Francisco, CA, USA, Nov. 2000.

[3] Liu,X, Ravindran,K, Liu,B, Loguinov,D, "Single-Hop Probing Asymptotics in Available Bandwidth Estimation: Sample-Path Analysis", Proc. ACM Internet Measurement Conference, 2004.

[4] Park, K-J, Lim,H, Choi,C-H, "Stochastic Analysis of Packet-Pair Probing for Network Bandwidth Estimation", Computer Networks, 50, 2006, pp. 1901-15.

[5] Tunnicliffe,M.J., Winnett,M, "An Approximate Stochastic Analysis of the Packet-Pair Probing Technique for Available Bandwidth Estimation", submitted to International J. of Comm. Systems, September 2007.

[6] Johnsson,A, Melander,B, Björkman,M, "Bandwidth Measurement in Wireless Networks", Proc. Mediterranean Ad Hoc Networking Workshop, Porquerolles, France, 2005.

[7] Lee,A.M. "Applied Queuing Theory", Macmillan, 1966, p.34.

[8] Pitts,J.M. Schormans,J.A. "Introduction to IP and ATM Design and Performance", Wiley, 2000, p.117.

[9] Ibid, pp.287-98. 